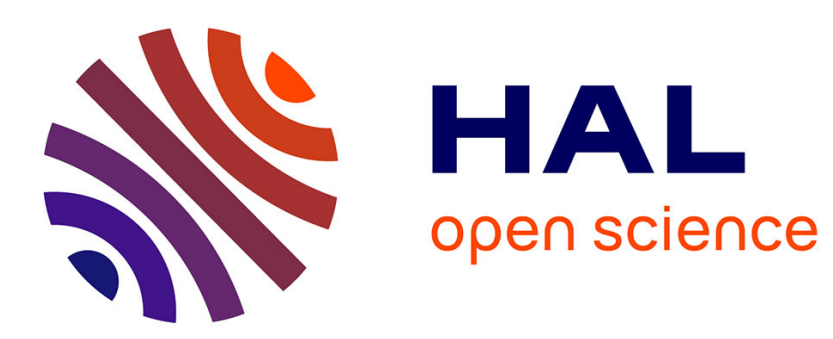

\title{
En Grèce, une crise migratoire chronique
}

Laurence Pillant

\section{To cite this version:}

Laurence Pillant. En Grèce, une crise migratoire chronique. Plein Droit, 2016, 111 (4), 10.3917/pld.111.0031. hal-01792268

\section{HAL Id: hal-01792268 \\ https://hal.science/hal-01792268}

Submitted on 15 May 2018

HAL is a multi-disciplinary open access archive for the deposit and dissemination of scientific research documents, whether they are published or not. The documents may come from teaching and research institutions in France or abroad, or from public or private research centers.
L'archive ouverte pluridisciplinaire HAL, est destinée au dépôt et à la diffusion de documents scientifiques de niveau recherche, publiés ou non, émanant des établissements d'enseignement et de recherche français ou étrangers, des laboratoires publics ou privés. 


\section{EN GRÈCE, UNE CRISE MIGRATOIRE CHRONIQUE}

Laurence Pillant

GISTI | « Plein droit »

2016/4 n $111 \mid$ pages 31 à 34

ISSN 0987-3260

Article disponible en ligne à l'adresse :

http://www.cairn.info/revue-plein-droit-2016-4-page-31.htm

\section{Pour citer cet article :}

Laurence Pillant, «En Grèce, une crise migratoire chronique », Plein droit 2016/4 $\left(\mathrm{n}^{\circ} 111\right)$, p. 31-34.

DOI 10.3917/pld.111.0031

Distribution électronique Cairn.info pour GISTI.

(c) GISTI. Tous droits réservés pour tous pays.

La reproduction ou représentation de cet article, notamment par photocopie, n'est autorisée que dans les limites des conditions générales d'utilisation du site ou, le cas échéant, des conditions générales de la licence souscrite par votre établissement. Toute autre reproduction ou représentation, en tout ou partie, sous quelque forme et de quelque manière que ce soit, est interdite sauf accord préalable et écrit de l'éditeur, en dehors des cas prévus par la législation en vigueur en France. Il est précisé que son stockage dans une base de données est également interdit. 


\author{
Pays d'émigration jusqu'au début des années 1990, la Grèce est devenue \\ un pays d'immigration avec l'arrivée des migrants albanais après la \\ chute du mur de Berlin. L'arsenal législatif mis en place alors, basé sur \\ l'expulsion et le contrôle aux frontières, ne peut répondre au phénomène \\ migratoire qui émerge dans les années 2000 , à savoir une migration de \\ transit. Répression et détention deviennent des modes de gestion des \\ flux migratoires, sous couvert d'une situation d'urgence... qui dure.
}

\title{
En Grèce, une crise migratoire chronique
}

Laurence Pillant, doctorante géographe, Aix-Marseille université, CNRS (laboratoire TELEMMe) et Harokopio University (Grèce)

Vingt-six ans après l'arrivée massive des Albanais en Grèce après la chute du mur de Berlin et la fin de la dictature d'Enver Hoxha, les migrations continuent, dans ce pays, à être gérées dans l'urgence. Si, depuis 2015, les arrivées de migrants à la frontière gréco-turque sont inédites par leur proportion, les mesures prises pour la « gestion » de cette situation par le gouvernement grec et l'Union européenne s'inscrivent dans une continuité historique de dissuasion et de répression de la migration.
Pays d'émigration jusqu'au début des années 1990, la Grèce devient un pays d'immigration avec l'arrivée de migrants albanais. Cette migration qui apparaît massive et soudaine entraîne la mise en place d'instruments législatifs permettant de contrôler les entrées et le séjour des étrangers présents sur le territoire.

\section{La dissuasion par l'expulsion}

La loi de 1929, encore en vigueur à l'époque, qui vise à réguler les départs de la Grèce et non les arrivées, est un cadre législatif « obsolète » dans la nouvelle configuration migratoire ${ }^{1}$. Réagissant à l'arrivée des Albanais et conformément aux injonctions européennes, le gouvernement adopte en 1991 une loi qui permet l'expulsion immédiate des étrangers en situation irrégulière et renforce le contrôle aux frontières. Cette loi pose les jalons sécuritaires de la politique migratoire grecque qui repose sur l'idée que la régulation de l'immigration est une affaire de maintien de l'ordre et de santé publique ${ }^{2}$. On observe une criminalisation des migrants qui franchissent les frontières clandestinement et leur « rejet $[. .$.$] vers les$ zones de non-droit social du travail informel ${ }^{3} \gg$. Bien que l'association d'enjeux sécuritaires aux politiques migratoires soit une tendance européenne, la Grèce est particulièrement précoce en la matière ${ }^{4}$. D'emblée, les Albanais sont vus comme des migrants économiques (metanastes) et non comme des réfugiés (prosfigues), ce qui entretient une vision négative de leur 
présence en Grèce et légitime le rapport de violence instauré par l'Etat à leur égard. Cette violence prend la forme d'« opérations balai » dans les grands centres urbains, qui s'accompagnent de reconduites à la frontière et font l'objet d'une importante médiatisation. On peut y lire la construction d'un « ennemi intime » qui passe par une nécessaire « clarification » de l'identité grecque 5 , fondamentale dans l'élaboration d'un système de contrôle basé sur la répression des Albanais puis de l'ensemble des étrangers. Dès 1991, le gouvernement grec fait de l'expulsion un outil majeur de réponse à la migration albanaise. Ce mécanisme constitue par ailleurs un outil de négociation entre les deux pays, un levier politique interne et une « arme électorale » dont les politiciens peuvent se saisir ${ }^{6}$. Enfin, ces expulsions sont facilitées par la proximité du pays de renvoi malgré l'absence ${ }^{7}$ d'accords de réadmis$\operatorname{sion}^{8}$.

À partir des années 1990, du fait de sa contiguïté avec des pays non inclus dans l'espace Schengen, la Grèce a bénéficié d'une aide importante de l'UE pour le financement d'équipements et la modernisation des structures destinés à la surveillance des frontières. Mais le phénomène migratoire qui émerge dans les années 2000 à la frontière gréco-turque, qui correspond à une migration de transit pour les ressortissants des pays du Moyen-Orient, d'Afrique et d'Asie, rend difficilement applicables les mécanismes d'expulsion mis en place précédemment, et un nouveau cadre législatif reposant sur l'enfermement voit le jour.

\section{La dissuasion par l'enfermement}

Ces arrivées nouvelles, présentées comme épisodiques, sont prises en charge à la fois par les autorités et la société civile. Du début des années 2000 jusqu'à 2010, on constate une nette augmentation des interpellations à la frontière gréco-turque (2 431 personnes en 2003 pour 52484 en 2010). Les discours divergent sur la teneur répressive du contrôle et de l'enfermement, mais le manque de cadre législatif clair est unanimement admis. Des lieux «d'accueil » sont partiellement ouverts (à Chios, Lesbos et dans le Dodécanèse) ou fermés (à Samos et dans la région d'Évros). La dimension « humanitaire » de ce premier accueil et l'ouverture partielle de certains lieux ne doivent pas occulter la mise à l'écart produite par leur enclavement géographique. Au nom de l'augmentation des flux, sous la pression des populations locales avoisinant les lieux, de la présence ou non de soutiens aux migrants, et des changements de législation, l'enfermement devient progressivement la principale réponse aux franchissements irréguliers des frontières.

Contrairement aux Albanais, les migrants qui arrivent à la frontière gréco-turque ne sont pas expulsables faute d'accords de réadmission entre la Grèce et les pays de provenance. Les accords signés avec la Turquie en 2001, peu effectifs, ne permettent pas le renvoi de toutes les personnes en voie d'expulsion. En vertu de la loi n 2901 de 2001, elles sont, par conséquent, systématiquement condamnées à une peine de prison. Cette loi permet cependant au procureur de décider de suspendre la condamnation pénale au profit d'un renvoi dans les plus brefs délais. Si l'expulsion n'est pas possible et s'il y a des soupçons de fuite, les étrangers doivent être placés en rétention puis dans des établissements spéciaux, pour une durée qui ne peut dépasser trois mois.
La plupart des migrants en sortent munis d'une obligation de quitter le territoire dans les 30 jours. Si, au début des années 2000, les premières arrivées ont pu surprendre, cela n'a plus été le cas par la suite. La pérennisation des dispositifs d'urgence mis en place à l'époque s'explique par leur usage politique et économique ${ }^{9}$ et conduit finalement à l'institutionnalisation de l'enfermement comme modalité de contrôle migratoire.

En 2003, le règlement européen « Dublin II » impose à la Grèce de renforcer le contrôle des sorties du territoire vers les autres pays de l'UE. Combiné à l'application du dispositif Schengen, il a pour effet de transformer la Grèce en «cul-de-sac » pour les migrants qui s'y trouvent confinés. En 2004, l'organisation des Jeux olympiques s'accompagne d'exigences sécuritaires particulières, auxquelles s'ajoutent les interventions en Méditerranée et autres coopérations liées à la création de l'agence Frontex. Dans ce contexte, l'adoption de la loi n 3386 de 2005, même si elle comportait des mesures visant à favoriser l'intégration des étrangers par la reconnaissance d'un droit au séjour, s'inscrit dans la continuité des lois précédentes. Elle ne clarifie pas pour autant les normes régissant les lieux d'enfermement. La conjonction des règles européennes, des injonctions internationales et des pratiques policières entraînent ainsi une répression systématique des migrants, et la détention reste une modalité de la gestion des flux. En 2005, six structures officielles de détention d'étrangers sont implantées à la frontière gréco-turque. Parce que ces lieux, maintes fois dénoncés par des ONG et des institutions internationales ${ }^{10}$, contreviennent aux standards européens et internationaux en matière de privation de liberté, le débat ne porte 
désormais plus sur la légitimité de l'enfermement mais sur les possibilités d'en améliorer les conditions.

L'apparition, avec la loi $n^{\circ} 3907 / 2011$ prise en application de la directive européenne « retour », des « centres de préexpulsion » augmente considérablement la capacité rétentionnaire du pays. Situés à Komotini, Xanthi, Corinthe et Agmydaleza, ils peuvent héberger entre 800 et 1000 personnes et sont alimentés par les interpellations massives survenues dans le cadre de l'opération «Xenios Zeus », qui rappellent les « opérations balai » des années 1990. Malgré leur spécialisation annoncée, ils sont à l'image de l'enfermement en Grèce: des lieux mixtes accueillant des demandeurs d'asile, des personnes considérées comme vulnérables et d'autres en instance d'expulsion. La nouvelle loi crée également des centres d'identification à la frontière - les ancêtres des hotspots - (notamment à Lesbos, à Évros et Filakio), généralement attenants ou confondus avec les lieux de rétention. À l'ensemble de ces mesures s'ajoute la construction d'un mur à la frontière gréco-turque terrestre (à Nea Vissa).

Alors que la Grèce est déjà connue pour ses faibles taux de reconnaissance du statut de réfugié, la réforme de 2011 renforce l'importance du premier entretien à la frontière dans les centres d'identification, dont le personnel, même s'il n'est pas toujours qualifié, dispose d'un large pouvoir discrétionnaire. In fine, on observe une augmentation du nombre d'étrangers reconnus réfugiés, aux dépens des autres, soumis aux politiques de renvoi ou de retour « volontaire », avec des périodes d'enfermement toujours plus longues. La transposition de la directive européenne « retour » représente à cet égard une régression dans la mesure où elle autorise la détention jusqu'à 18 mois, cette durée pouvant encore être prorogée ${ }^{11}$.

Pour respecter les normes européennes et internationales, le «tri » opéré entre « réfugiés » et « non-réfugiés » devait permettre d'extraire les demandeurs d'asile des procédures de renvois et d'enfermement: c'est loin d'être le cas. Et si l'arrivée de Syriza au gouvernement a pu faire espérer la fermeture des lieux de détention d'étrangers, comme l'annonçait le programme d'Alexis Tsipras, et un meilleur accueil des migrants, les arrivées importantes de 2015 vont bouleverser ces perspectives.

\section{La demande d'asile: un nouvel outil répressif}

Qualifiée de « crise migratoire » sans précédent, cette augmentation est une nouvelle fois perçue comme une situation d'urgence et gérée de la même façon qu'au début des années 2000. Puisqu'il s'agit d'accueil d'urgence, les ONG se pressent sur les îles de la mer Égée où des milliers de personnes débarquent chaque jour à partir du printemps 2015. Pour les désengorger, l'État finance le transfert des migrants vers Athènes. Devant l'ampleur du phénomène et la saturation des services de premier accueil, l'enfermement n'est plus possible. Très vite, l'enjeu est double: faire transiter les personnes tout en les identifiant.

Durant quelques mois, les frontières balkaniques deviennent aisément franchissables, et beaucoup de personnes «bloquées » en Grèce depuis des années en profitent pour en partir. Pendant ce temps, ce qu'on qualifie de « traversée de la mort », entre Turquie et Grèce, tue chaque jour et met des milliers de personnes en danger. Avec le retentissement médiatique et l'usage politique de cette crise, la « route balkanique $»^{12}$ va peu à peu se refermer, prenant au piège les migrants en Grèce. La fermeture de la frontière entre la Grèce et la Macédoine marque le début du blocage des exilés à Idomeni, à Athènes et sur les îles. Partout, la circulation est ralentie jusqu'à l'arrêt et le confinement de chacun où il se trouve: la Grèce est de plus en plus isolée du reste de l'Europe et menacée par ses partenaires européens d'une sortie de l'espace Schengen.

Le processus de « relocalisation » (qui prévoit le transfert, depuis la Grèce et l'Italie, de personnes jugées éligibles à l'asile dans d'autres pays de l'UE), lancé en septembre 2015 par l'UE, fonctionne très mal (voir article p. 3). Outils de la « relocalisation », les hotspots font petit à petit leur apparition dans le paysage frontalier du sud de l'Europe. Regroupés sous la même appellation sans valeur juridique, ils recouvrent des réalités bien différentes. En Grèce, où le premier hotspot voit le jour en octobre 2015 sur l'île de Lesbos, à Moria, ce sont des « centres d'identification et de réception » au sein desquels les étrangers peuvent être privés de liberté pour une durée qui ne peut excéder 25 jours. Présentés comme des lieux d'accueil et de « prise en charge », ils vont bientôt devenir des nasses pour les migrants qui y sont retenus.

La conclusion d'un accord entre l'UE et la Turquie, au mois de mars 2016, constitue un tournant dramatique pour des milliers de personnes bloqués sur les îles de la mer Égée: aux mécanismes d'expulsion et d'enfermement s'ajoute alors le chantage à l'asile. Combiné à la modification concomitante de la loi sur l'asile, qui permet aux autorités grecques de refuser l'octroi d'une protection au motif que le demandeur a transité par un « premier pays d'asile », le 
mécanisme introduit par l'accord UE-Turquie ne contrevient formellement pas au principe de nonrefoulement posé par la convention de Genève sur les réfugiés: toutes les demandes d'asile déposées sont examinées, mais, même pour les personnes jugées éligibles à une protection, une décision de refus d'admission sur le territoire grec peut être prise s'il y a eu transit par «un pays où le requérant est considéré comme réfugié, ou profite d'une protection effective, incluant le bénéfice de jouir du principe de nonrefoulement » et dont il ne peut pas être refoulé ${ }^{13}$. La Turquie répond à ces critères. Dans ce cas, une procédure de réadmission peut-être lancée. En pratique, le caractère quasi systématique des refus d'admission des demandeurs d'asile dans les hotspots traduit les objectifs clairement assumés de l'accord UE-Turquie: il s'agit de dissuader les migrants de tenter de rejoindre par la mer les îles grecques, donc l'UE, en confiant à la Turquie le soin de les prendre en charge.

\section{«Confinement insulaire »}

Le coup d'État de juillet 2016 et la répression sévère qui l'a suivi en Turquie ont eu pour effet indirect de geler l'application de l'accord UE-Turquie. Mais la menace d'expulsion pèse sur la grande majorité des personnes présentes dans les hotspots grecs. On peut donc parler de « confinement insulaire ${ }^{14} \gg$ sur les îles de la mer Égée où plus de 13000 migrants (pour une capacité de 7450 places) sont laissés dans l'incertitude du sort qui leur sera réservé. L'effet dissuasif est particulièrement fort.

Le gouvernement d'Alexis Tsipras n'a de cesse de réclamer plus de moyens, mais la faible confiance en la Grèce, fruit d'années de dénigrement du pays, couplée à une gestion des fonds par appels d'offres et projets, entraîne un financement direct de l'UE aux organisations internationales qui disposent ainsi d'un droit de regard sur les fonctions régaliennes. Cette privatisation des structures d'accueil de migrants fragilise le gouvernement qui peine à coordonner l'ensemble des lieux.

Mais les arrivées se tarissent; la dissuasion par la menace des renvois semble agir en faveur d'un contournement de la voie turco-grecque par les migrants. L'ensemble des mesures prises institutionnalise la Grèce comme un lieu de tri et d'« accueil » des migrants, et les îles de la mer Égée comme une zone d'enfermement soumise à des enjeux nationaux et internationaux qui ne concernent pas uniquement la migration et mettent en scène une politique du rejet, de la peur et de la dissuasion. Sur le continent, les ONG mâ̂trisent de bout en bout la gestion des lieux d'accueil. La question des droits des migrants se pose avec force, notamment en matière de scolarisation et d'accès à la santé, créant des tensions dans un pays ravagé par des années de crise économique. Si la posture du gouvernement contraste avec la « traditionnelle » criminalisation des étrangers véhiculée par les pouvoirs publics et les médias, les moyens manquent et les pressions sont fortes. Fin 2016, plus de 60000 réfugiés étaient abandonnés par l'Europe en Grèce, sans autre perspective que de voir leur nombre encore augmenter avec la menace d'une reprise des « renvois Dublin ${ }^{15}$ par les autres États membres de l'UE. Une situation insoutenable à bien des égards, en premier lieu pour les migrants euxmêmes.

\footnotetext{
1 Anastassia Tsoukala, « Le contrôle de l'immigration en Grèce dans les années 1990 », Cultures Q
}

Conflits, n 26-27, 1997 ; Pierre Sintès, La raison du mouvement: territoires et réseaux de migrants albanais en Grèce, Karthala, 2010.

2 II y est spécifié que le législateur refuse l'entrée à tout étranger atteint de maladie considérée comme susceptible de mettre en jeu la santé publique; la loi précédente prévoyait le refus d'entrée à tout étranger atteint d'une maladie mentale...

3 Pierre Sintès, ibid.

4 Evelyne Ritaine, «La fabrique politique d'une frontière européenne en Méditerranée. Le "jeu du mistigri" entre les États et l'Union », Les études du CERI, 2012.

5 Pierre Sintès, ibid.

6 Pierre Sintès, « La migration des Albanais en Grèce. Difficultés méthodologiques pour une étude géographique », Revue européenne des migrations internationales, vol. $17, \mathrm{n}^{\circ} 3,2001$, p. $67-85$.

${ }^{7}$ Accord de réadmission signé en 2005 entre l'UE et l'Albanie.

${ }^{8}$ La Grèce a signé plusieurs accords de réadmission : voir la liste sur www.migreurop.org/ article1591.html

9 Pierre Sintès, « Manœuvres politiques et discours de l'urgence: la frontière égéenne de la Grèce au cœur d'enjeux nationaux et européens », in N. Ribas-Mateos (dir.), El Rio Bravo Mediterráneo. Las Regiones Fronterizas en la época de la Globalización, Barcelone, 2011, p. 375-390; Louise Tassin, «Le mirage des hotspots », Savoir/ Agir, vol. 36, $\mathrm{n}^{\circ}$ 2, 2016, p. 39-45.

10 Dont le Comité européen pour la prévention de la torture et la Commission nationale grecque pour les droits de l'Homme.

"Selon cet avis du 20 mars 2014, le placement en détention peut être illimité si l'étranger refuse de coopérer avec les autorités grecques

12 Les frontières de la Hongrie se ferment en août 2015, celles de la Macédoine, de l'Autriche et de la Slovénie en novembre 2015.

${ }^{13}$ Traduction libre de l'article 54 de la loi 4375/2016.

14 Karen Akoka et Olivier Clochard, « Régime de confinement et gestion des migrations sur l'île de Chypre », L'Espace politique. Revue en ligne de géographie politique et de géopolitique, $\mathrm{n}^{\circ} 25,2015$.

${ }^{15}$ Le règlement européen « Dublin III » permet aux États de l'UE de renvoyer les demandeurs d'asile dans le premier pays par lequel ils sont arrivés en Europe. Depuis 2011, les transferts vers la Grèce ont été interrompus à la suite de deux arrêts de la Cour européenne des droits de l'Homme et de la Cour de justice de l'Union européenne qui ont mis en évidence des défaillances systémiques dans le système d'asile grec. En juin 2016, la Commission européenne a adopté une recommandation en vue de rétablir les transferts vers la Grèce du fait « des efforts continus [déployés par le pays] pour améliorer son système d'asile ». 